\title{
Erratum: Optimizing for an arbitrary perfect entangler. II. Application [Phys. Rev. A 91, 062307 (2015)]
}

\author{
Michael H. Goerz, Giulia Gualdi, Daniel M. Reich, Christiane P. Koch, Felix Motzoi, K. Birgitta Whaley, \\ Jiří Vala, Matthias M. Müller, Simone Montangero, and Tommaso Calarco
}

(Received 7 January 2019; published 23 January 2019)

DOI: 10.1103/PhysRevA.99.019903

Equations (26) and (27) of our article contain a typographical error and should read

$$
\begin{aligned}
J_{\mathrm{LI}}^{\prime}(\tilde{U}) & =w J_{\mathrm{LI}}(\tilde{U})+(w-1)\left(1-\frac{1}{4} \operatorname{tr}\left[\tilde{U}^{\dagger} \tilde{U}\right]\right), \\
\mathcal{D}^{\prime}(\tilde{U}) & =w \mathcal{D}(\tilde{U})+(w-1)\left(1-\frac{1}{4} \operatorname{tr}\left[\tilde{U}^{\dagger} \tilde{U}\right]\right) .
\end{aligned}
$$

That is, in the first term in each equation, the "base functional" is evaluated for the projection $\tilde{U}$, which is nonunitary in general, and not its closest unitary $U$. We have also added primes on the left-hand sides to distinguish them from the base functionals. The correct equations were used in obtaining the results presented in Sec. V of the article; therefore, the error does not affect our conclusions.

The equations for the local invariants $\left(g_{1}, g_{2}, g_{3}\right)$ that enter Eqs. (26) and (27) are defined in Ref. [1] for arguments in SU(4). For general $\tilde{U}$, the appropriate expressions are

$$
g_{1}=\operatorname{Re}\left\{\frac{1}{16 \operatorname{det} \tilde{U}} \operatorname{tr}^{2}(m)\right\}, \quad g_{2}=\operatorname{Im}\left\{\frac{1}{16 \operatorname{det} \tilde{U}} \operatorname{tr}^{2}(m)\right\}, \quad g_{3}=\operatorname{Re}\left\{\frac{1}{4 \operatorname{det} \tilde{U}}\left[\operatorname{tr}^{2}(m)-\operatorname{tr}\left(m^{2}\right)\right]\right\},
$$

with $m=U_{\mathrm{B}}^{T} U_{\mathrm{B}}, U_{\mathrm{B}}=Q^{\dagger} \tilde{U} Q$, and

$$
Q=\frac{1}{\sqrt{2}}\left(\begin{array}{rrrr}
1 & 0 & 0 & i \\
0 & i & 1 & 0 \\
0 & i & -1 & 0 \\
1 & 0 & 0 & -i
\end{array}\right)
$$

The use of $\tilde{U}$ instead of $U$ in the correct Eqs. (26) and (27) is due to the fact that, for Krotov's method, the derivative of the functional with respect to the states must be known analytically, see Eq. (33b) in the article. As the transformation $\tilde{U} \rightarrow U$ involves a singular value decomposition, which is nonanalytical, it is not possible to calculate the necessary derivatives for a functional that contains $J_{\mathrm{LI}}(U)$ or $\mathcal{D}(U)$.

The more nonunitary $\tilde{U}$ is, the less $J_{\mathrm{LI}}(\tilde{U})$ and $\mathcal{D}(\tilde{U})$ give any information regarding the progress of an optimization towards its actual objective, as the local invariants are well defined only in the unitary regime. Thus, the weight $w$ in Eqs. (26) and (27) must be chosen to balance the two terms of the functional. As pointed out in the article, $w$ can be changed adaptively during the optimization. Initially, a lower value for $w$ puts a greater emphasis on pushing $\tilde{U}$ towards being unitary, so that $J_{\mathrm{LI}}(\tilde{U})$ and $\mathcal{D}(\tilde{U})$ become informative. Then, in later iterations, $w$ can be increased to put more emphasis on actually reaching the desired equivalence class of a gate, respectively a perfect entangler.

The authors would like to thank D. Basilewitsch for bringing the error to their attention.

[1] P. Watts, J. Vala, M. M. Müller, T. Calarco, K. B. Whaley, D. M. Reich, M. H. Goerz, and C. P. Koch, Phys. Rev. A 91, 062306 (2015). 